Article

\title{
Luminal STIM1 Mutants that Cause Tubular Aggregate Myopathy Promote Autophagic Processes
}

\author{
Matthias Sallinger ${ }^{1}$, Adéla Tiffner ${ }^{1}$, Tony Schmidt ${ }^{2}$, Daniel Bonhenry ${ }^{3}$, Linda Waldherr ${ }^{2}(\mathbb{D}$, \\ Irene Frischauf ${ }^{1}$, Victoria Lunz ${ }^{1}{ }^{1}$, Isabella Derler ${ }^{1}$, Romana Schober ${ }^{1,2, *}$ and \\ Rainer Schindl 2,4,*(D) \\ 1 Institute of Biophysics, JKU Life Science Center, Johannes Kepler University Linz, A-4020 Linz, Austria; \\ matthias.sallinger@jku.at (M.S.); adela.tiffner@jku.at (A.T.); irene.frischauf@jku.at (I.F.); \\ vici.lunz@gmail.com (V.L.); isabella.derler@jku.at (I.D.) \\ 2 Gottfried Schatz Research Center, Medical University of Graz, A-8010 Graz, Austria; \\ tony.schmidt@medunigraz.at (T.S.); linda.waldherr@medunigraz.at (L.W.) \\ 3 Center for Nanobiology and Structural Biology, Institute of Microbiology, Academy of Sciences of the Czech \\ Republic, CZ-373 33 Nove Hrady, Czech Republic; bonhenry@nh.cas.cz \\ 4 BioTechMed-Graz, A-8010 Graz, Austria \\ * Correspondence: romana.schober@jku.at (R.S.); rainer.schindl@medunigraz.at (R.S.)
}

Received: 3 June 2020; Accepted: 19 June 2020; Published: 21 June 2020

\begin{abstract}
Stromal interaction molecule 1 (STIM1) is a ubiquitously expressed $\mathrm{Ca}^{2+}$ sensor protein that induces permeation of Orai $\mathrm{Ca}^{2+}$ channels upon endoplasmic reticulum $\mathrm{Ca}^{2+}$-store depletion. A drop in luminal $\mathrm{Ca}^{2+}$ causes partial unfolding of the N-terminal STIM1 domains and thus initial STIM1 activation. We compared the STIM1 structure upon $\mathrm{Ca}^{2+}$ depletion from our molecular dynamics (MD) simulations with a recent 2D NMR structure. Simulation- and structure-based results showed unfolding of two $\alpha$-helices in the canonical and in the non-canonical EF-hand. Further, we structurally and functionally evaluated mutations in the non-canonical EF-hand that have been shown to cause tubular aggregate myopathy. We found these mutations to cause full constitutive activation of $\mathrm{Ca}^{2+}$-release-activated $\mathrm{Ca}^{2+}$ currents $\left(\mathrm{I}_{\mathrm{CRAC}}\right)$ and to promote autophagic processes. Specifically, heterologously expressed STIM1 mutations in the non-canonical EF-hand promoted translocation of the autophagy transcription factors microphthalmia-associated transcription factor (MITF) and transcription factor EB (TFEB) into the nucleus. These STIM1 mutations additionally stimulated an enhanced production of autophagosomes. In summary, mutations in STIM1 that cause structural unfolding promoted $\mathrm{Ca}^{2+}$ down-stream activation of autophagic processes.
\end{abstract}

Keywords: STIM; Orai; $\mathrm{Ca}^{2+}$; SOCE; MITF; TFEB; hydrophobic pocket; EF-hand; tubular aggregate myopathy

\section{Introduction}

$\mathrm{Ca}^{2+}$ entry via store-operated $\mathrm{Ca}^{2+}$ channels (SOCs) provides an essential signaling mechanism for cellular immune responses and is required to control the filling state of the endoplasmic reticulum (ER) in virtually every cell type [1-4]. Activation of this sustained SOC entry (SOCE) is provided on a molecular basis by the ER $\mathrm{Ca}^{2+}$-sensing proteins stromal interaction molecule 1 (STIM1) and STIM2 and the plasma membrane (PM) $\mathrm{Ca}^{2+}$ channels Orai1, 2, and 3 [5-9]. STIM1 senses luminal $\mathrm{Ca}^{2+}$ with a canonical EF-hand and responds to a reduced $\mathrm{Ca}^{2+}$ concentration within the ER $[10,11]$. Resting $\mathrm{Ca}^{2+}$ concentrations are at around $800 \mu \mathrm{M}$, while a release of ER $\mathrm{Ca}^{2+}$, physiologically mediated by inositol-3-phosphate or ryanodine receptors, leads to a drop in ER $\mathrm{Ca}^{2+}$ concentrations to 100-400 $\mu \mathrm{M}[12,13]$. As a consequence, STIM1 loses bound $\mathrm{Ca}^{2+}$ within its canonical EF-hand [12,14]. 
Functionally, this $\mathrm{Ca}^{2+}$-binding is important to keep the luminal STIM1 structure folded [15-17]. $\mathrm{Ca}^{2+}$-free conditions partially destabilize the luminal STIM1 and result preferentially in STIM1 dimers [11,15]. Even larger aggregates arise from C-terminal STIM1 multimerization [18,19]. However, the STIM C-terminus is not only responsible for STIM clustering, but also extends and binds directly to the Orai1 channel [20-27] at specifically localized ER-PM junctions, where the membranes are only 10 to $17 \mathrm{~nm}$ apart $[12,18,28]$. The STIM/Orai complexes are stable over long time periods to mediate $\mathrm{Ca}^{2+}$ signaling for several minutes up to hours [29]. $\mathrm{Ca}^{2+}$ influx initiates local signaling cascades, such as the well-characterized activation of the nuclear factor of activated T-cells (NFAT) [4,30-33].

Thus, luminal STIM1 $\mathrm{Ca}^{2+}$-dependent unfolding is a key prerequisite for the activation of a precisely controlled SOCE signaling cascade. The luminal structures of STIM1 and STIM2 have been determined and show a canonical and a non-canonical EF-hand and a sterile alpha motif (SAM) domain (Figure 1A) $[11,17,34]$. In the $\mathrm{Ca}^{2+}$-bound state of STIM, all three domains are tightly packed together [11,17]. NMR structures determined a hydrophobic pocket formed by amino acids in the canonical, the non-canonical EF-hand, and the SAM domain $[11,17,34]$. These hydrophobic pocket residues in human STIM1 are V68, I71, H72, M75, L92, L96, and K104 within the canonical EF-hand, F108, H109, I115, and L120 in the non-canonical EF-hand, and the residues L195 and L199 in the SAM domain. It is of great interest that mutations in several of these hydrophobic pocket residues within the canonical (H72Q and L96V) and non-canonical (F108I, H109N, H109R, and I115F) EF-hands result in tubular aggregate myopathy $[35,36]$. In line with this finding, mutations that interfere with the hydrophobic pocket within the canonical EF-hand (H72R or H72Q, L92P, L96V), the non-canonical EF-hand (F108I, F108D-G110D, H109N or H109R and I115F), and the SAM domain (L195R) result in aggregation, constitutively active $\mathrm{Ca}^{2+}$ entry, or NFAT activation $[11,15,35,36]$.

In this research, we investigate whether these non-canonical EF-hand mutations stimulate $\mathrm{Ca}^{2+}$-mediated signaling pathways in autophagy and examined transcriptional processes and autophagosome formation. We therefore focused our attention on the calcium-dependent transcription factor EB (TFEB) and its close homolog, the microphthalmia-associated transcription factor (MITF). Both transcription factors are part of the MIT/TFE family and are master regulators of lysosomal biogenesis, autophagy, mitophagy, and stress response [37-40]. Activation of TFEB is mediated by the $\mathrm{Ca}^{2+} /$ Calmodulin-dependent phosphatase Calcineurin (CaN) [41]. Dephosphorylation of TFEB controls the translocation of this transcription factor to the nucleus [41,42]. This CaN-dependent phosphorylation is exquisitely similar to STIM1/Orai1-mediated translocation of NFAT [32,33], and previous research has already associated SOCE with TFEB activation in pancreatic acinar cells [43]. The rise in $\mathrm{Ca}^{2+}$ concentration responsible for TFEB activation originates either from SOCE or from lysosomal store depletion $[41,43,44]$.

Specifically, in the resting state, TFEB and MITF are phosphorylated at multiple sites, which is a prerequisite for 14-3-3 binding [45]. The 14-3-3 protein is responsible for cytosolic sequestration, possibly due to the masking of a nuclear localization signal (NLS) [42]. Hence, CaN dephosphorylates TFEB, which allows its nuclear translocation and activates expression of autophagy genes [41]. TFEB activity can be linked specifically to autophagosome promotion, as autophagosome formation is reduced upon silencing of $\mathrm{CaN}[41,43,46]$. This identifies $\mathrm{CaN}$ as a regulator of the autophagic pathway [41]. However, autophagosome formation can also take place in a transcription-independent manner, hence in a TFEB-independent manner, as lysosomal $\mathrm{Ca}^{2+}$-store depletion via transient receptor potential mucolipin 1 (TRPML1) is sufficient to induce autophagosome biogenesis [47].

Here, we link tubular aggregate myopathy (TAM) mutations in STIM1 to their role in destabilizing the hydrophobic pocket and in promoting autophagic pathways. Specifically, we found that mutations of hydrophobic residues that form the non-canonical EF-hand result in constitutively active $\mathrm{Ca}^{2+}$ influx. In addition, constitutively active STIM1 mutants of both the non-canonical and the canonical EF-hand induced autophagic processes, including activation of the calcium-dependent transcription factors MITF and TFEB. Furthermore, we determined enhanced autophagosome formation upon overexpression of constitutively active STIM mutants. 


\section{Results}

\subsection{The Hydrophobic Pocket of STIM1 Unfolds upon $\mathrm{Ca}^{2+}$ Depletion or Due to a Tubular Aggregate Myopathy Mutation}

We initially used molecular dynamics (MD) simulations to investigate how the resting-state luminal STIM1 (Figure 1A,B) rearranges to reach its $\mathrm{Ca}^{2+}$-depleted conformation (Figure $1 \mathrm{C}$ ). We visualized the helices of the NMR structure of STIM1 in its $\mathrm{Ca}^{2+}$-bound resting state as cylinders (Figure 1A-C) [11]: the canonical (helices 1 and 2) and the non-canonical (helices 3 and 4) EF-hands are connected via the short helix 5 to the SAM domain (helices 6 to 10) (Figure 1A,B and [11]). The helix nomenclature is in accordance with that in previous research [11]. MD simulations revealed that, in the $\mathrm{Ca}^{2+}$-depleted state, only helices 1 and 4 of the canonical and non-canonical EF-hands, respectively, are maintained (Figure 1C). Helices 2 and 3 exhibit a large unfolded loop (Figure 1C, lower panel). In agreement with our MD simulations, helices 2 and 3 remained unassigned in the 2D NMR, which suggests an unfolded secondary structure [17]. Furthermore, in line with our results, helices 1 and 4 are maintained, and the SAM domain (helices 6 to 10) together with the connecting helix 5 remain folded both in 2D NMR and in MD simulations [17].

A

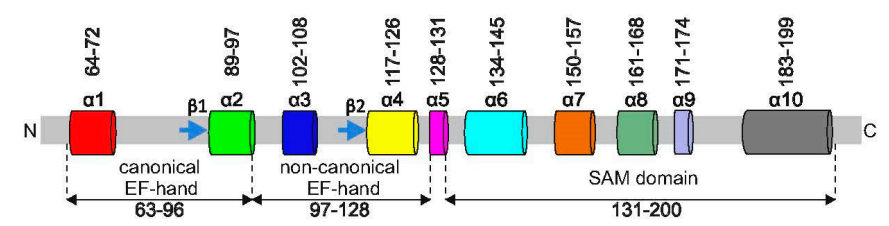

B
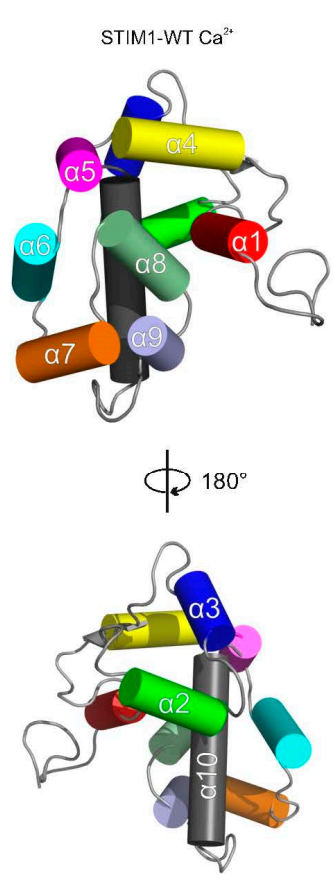

C
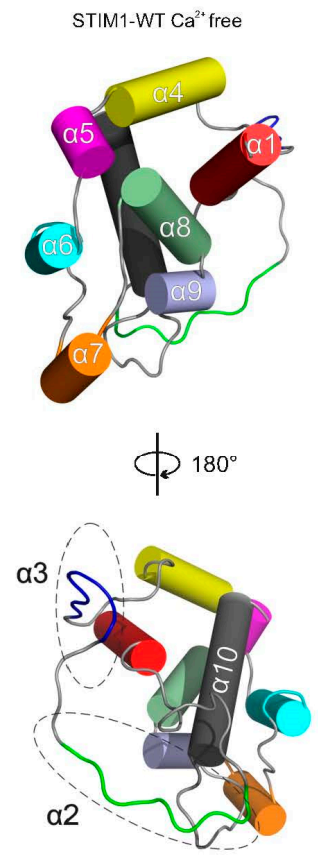

Figure 1. Conformational rearrangement of luminal STIM1 upon $\mathrm{Ca}^{2+}$ depletion. (A) Schematic representation of the secondary structure of the canonical, non-canonical, and sterile alpha motif (SAM) domains in luminal STIM1 proteins ( $\alpha$-helices are shown as cylinders and $\beta$-sheets as arrows). (B) Two orientations are shown for the $\mathrm{Ca}^{2+}$-bound resting state of STIM1 based on NMR results [11]. The helices (shown as cylinders) are labeled as follows: canonical EF-hand: helices 1 (red) and 2 (green); non-canonical EF-hand: helices 3 (blue) and 4 (yellow); a short connecting helix 5 (purple); and the SAM domain: helices 6 to 10 (turquoise, brown, dark green, light blue, gray). (C) Luminal STIM1 structure (from MD simulations) upon $\mathrm{Ca}^{2+}$ depletion compared with (B) in terms of helix structures. Helices 2 and 3, indicated by dashed circles, are unfolded in MD simulations. 
We investigated STIM1 residues that are associated with tubular aggregate myopathy $[35,36]$ when mutated (F108I, H109N/R, I115F; Figure 2A, lower panel). These residues are located within the non-canonical EF-hand of STIM1 and form part of the hydrophobic pocket (Figure 2A, upper panel). The hydrophobic pocket residues are tightly packed together in the center of the folded STIM1 structure and keep the resting STIM1 folded (Figure 2B) [11,15]. The greatest reorientation in hydrophobic canonical EF-hand residues upon transition from the resting to the active state is visible for L92 and L96. Both residues are substantially separated from the core of the STIM1 structure under $\mathrm{Ca}^{2+}$-free conditions (Figure 2C). Previous results have already highlighted the importance of these two leucines, and their mutation results in constitutive STIM1 activation [15]. Two non-canonical EF-hand residues, F108 and H109, are also detached from the remaining core of the hydrophobic pocket (Figure 2C).

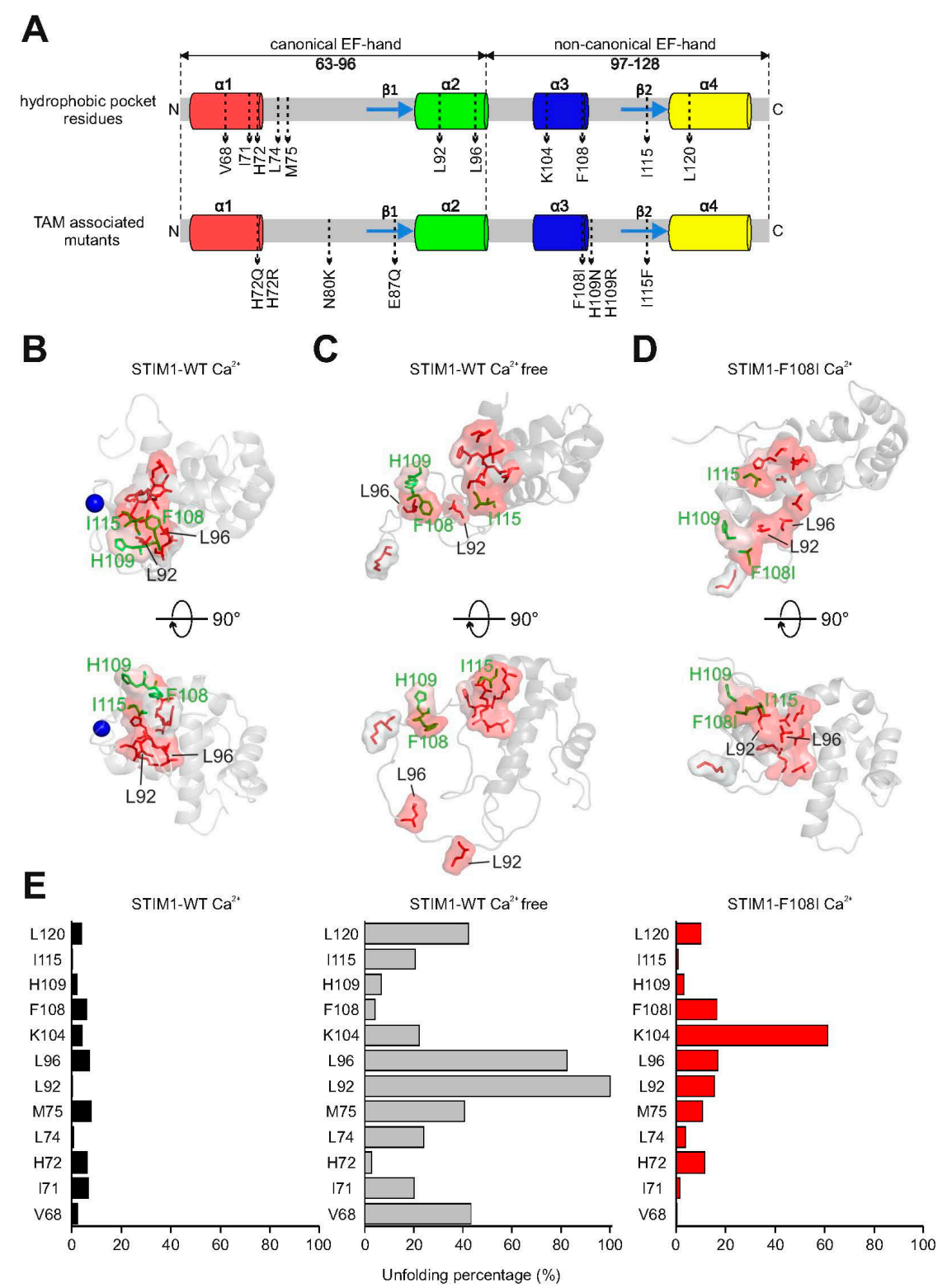

Figure 2. Unfolding of the hydrophobic pocket of STIM1 upon $\mathrm{Ca}^{2+}$ depletion. (A) Schematic representation of canonical and non-canonical EF-hand structures showing $\alpha$-helices as cylinders and $\beta$-sheets as arrows. In the upper panel, specific amino acids that form the hydrophobic pocket (as described in [11]) are highlighted. The lower panel shows disease-related mutations that are located in the canonical and non-canonical EF-hand domains [35,36]. (B-D) Hydrophobic pocket residues from 
panel (A) are shown both as red spheres and as stick models in two orientations, $(\mathbf{B})$ in the $\mathrm{Ca}^{2+}$-bound resting state of STIM1 based on NMR results and a bound $\mathrm{Ca}^{2+}$ ion (shown as blue sphere) [11], (C) upon $\mathrm{Ca}^{2+}$ depletion or (D) in a STIM1-F108I mutant (from MD simulations [17]). In addition, hydrophobic residues of the non-canonical EF-hand are shown as green sticks. (E) Relative proportion of unfolding (in \%) for residues of the canonical and non-canonical EF-hands that contribute to the hydrophobic pocket. The level of unfolding was compared to that of the L92 residue, which showed the highest level of unfolding and was set to $100 \%$.

An F108I mutation, found in patients with tubular aggregate myopathy, causes constitutive $\mathrm{Ca}^{2+}$ signaling [15], so we analyzed it further in MD simulations. STIM1-F108I mutation yielded unfolding of helix 3 (Figure S1B), which includes hydrophobic pocket residues K104, F108, and H109 (Figure 2D). In the simulations, these residues are separated from the rest of the hydrophobic pocket. All other luminal helices remain intact due to the presence of stabilizing $\mathrm{Ca}^{2+}$ ions (Figure $\left.\mathrm{S} 1 \mathrm{~A}, \mathrm{~B}\right)$. This accords with the finding that purified STIM1-F108I fragments unfold earlier in temperature-dependent measurements [15]. Next, we quantified to which extent each hydrophobic pocket residue is unfolded in (i) the $\mathrm{Ca}^{2+}$-free STIM1 structure and (ii) the STIM1-F108I mutation. We evaluated all hydrophobic pocket residues of the canonical and non-canonical EF-hands for their relative mean distance to the center of the EF-hands in the last $100 \mathrm{~ns}$ of the MD simulations (Figure 2E). As expected from the visualization of the $\mathrm{Ca}^{2+}$-free, unfolded STIM1 structure, L92 and L96 showed a high degree of unfolding, while the rest of the canonical and non-canonical EF-hands was only partially unfolded (Figure 2E; middle) [15]. In the STIM1-F108I simulation, K104 and I108 exhibited the highest degree of unfolding. The amount of unfolding of all hydrophobic residues was in general higher for the $\mathrm{Ca}^{2+}$-free STIM1 than for STIM1-F108I.

\subsection{Puncta Formation and Constitutive $I_{C R A C}$ Currents Are Induced by STIM1 Hydrophobic Pocket TAM Mutations}

We additionally investigated STIM1 hydrophobic-pocket TAM mutants at the cellular level and selected single-point mutations in the non-canonical EF-hand (F108I, H109N, H109R, and I115F) for live-cell experiments. First, we evaluated the localization of YFP-tagged STIM1 wild type and mutants upon overexpression in HEK cells. Wild type STIM1 exhibited tubular localization in the resting state [48] (Figure 3A, upper panel). ER Ca ${ }^{2+}$-store depletion was induced by treatment with thapsigargin (TG, $1 \mu \mathrm{M}, 5 \mathrm{~min}$ ), an inhibitor of Sarcoplasmic/endoplasmic reticulum calcium ATPase (SERCA) pumps. Consequently, wild type STIM1 rearranged and exhibited puncta formation (Figure 3A, lower panel). In contrast, STIM1 non-canonical EF-hand mutants (F108I, H109N, H109R, and I115F) already exhibited pre-clustered localization under resting conditions, similarly to STIM1 wild type upon ER store depletion (Figure 3B). Addition of TG did not further increase clustering of these STIM1 mutants, which indicates that these mutations switch STIM1 into a constitutively active state. These experiments are very well in line with our recent MD simulations on STIM1-F108I that loses dynamic $\mathrm{Ca}^{2+}$ interactions of the canonical EF-hand over time [15].

To evaluate whether these STIM1 hydrophobic pocket mutants are able to fully activate endogenous store-operated channels, we performed whole-cell patch-clamp experiments in rat basophilic leukemia (RBL) cells. This cell type has been very well described for large $\mathrm{Ca}^{2+}$-release-activated $\mathrm{Ca}^{2+}$ currents $\left(\mathrm{I}_{\mathrm{CRAC}}\right)[49,50]$. Store depletion for the activation of $\mathrm{Ca}^{2+}$ signaling of STIM1 wild type cells was induced by EGTA in the patch pipette. EGTA complexes $\mathrm{Ca}^{2+}$ ions with high affinity and results in delayed passive ER $\mathrm{Ca}^{2+}$-store depletion. Overexpression of wild type STIM1, therefore, resulted in delayed activation and reached a maximum $\mathrm{I}_{\mathrm{CRAC}}$ after $200 \mathrm{~s}$ (Figure 3C). In contrast, each of the overexpressed STIM1 non-canonical EF-hand mutants (F108I, H109N, H109, and I115F) exhibited robust constitutively active $\mathrm{I}_{\mathrm{CRAC}}$ immediately after whole-cell break-in (Figure 3C,D). The current/voltage relationship of maximum currents revealed a similar inward-rectification of $\mathrm{I}_{\text {CRAC }}$ generated by STIM1 wild type and the mutants (Figure 3E). 
A

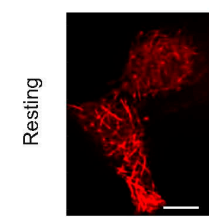

STIM1-WT

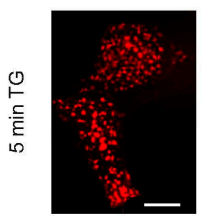

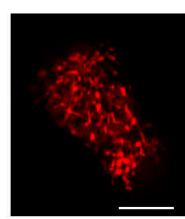

STIM1-F108I

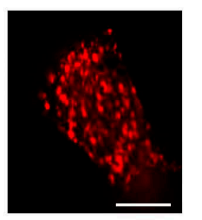

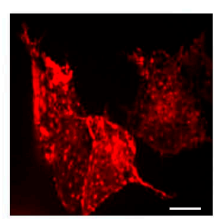

STIM1-H109N

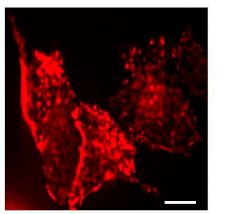

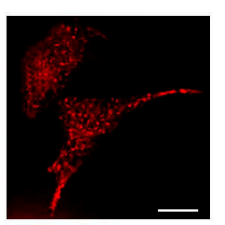

STIM1-H109R

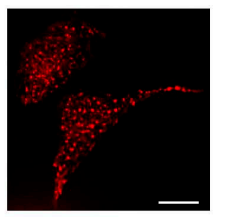

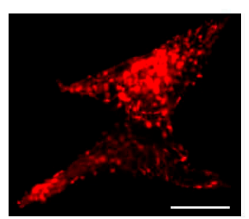

STIM1-I115F

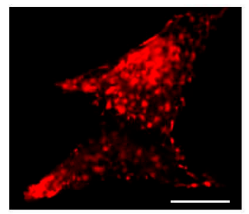

B

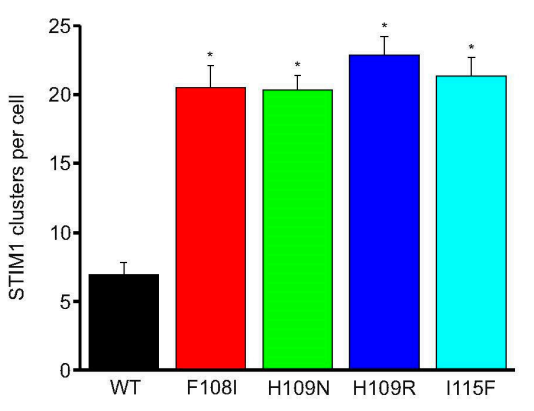

D

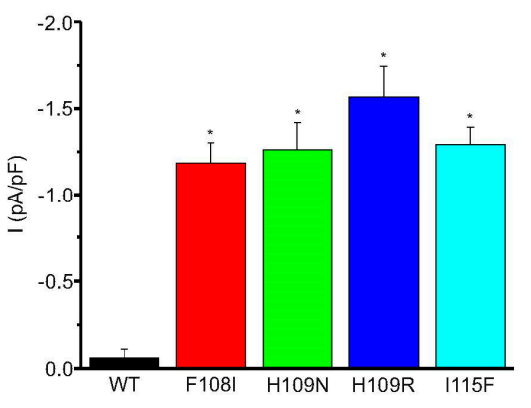

C

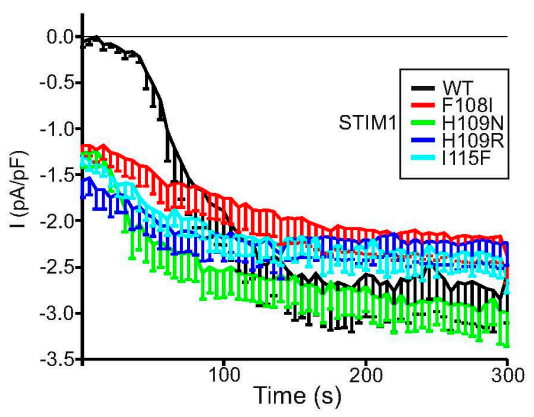

$\mathbf{E}$

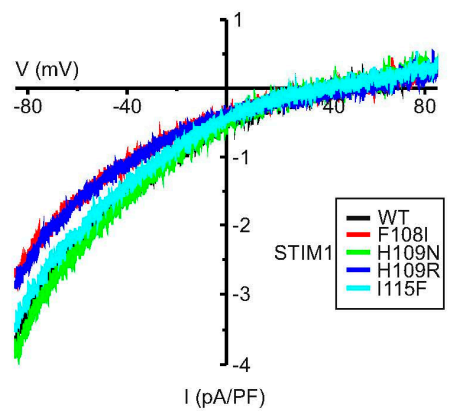

Figure 3. STIM1 non-canonical EF-hand mutants constitutively activate CRAC channel currents. (A) Representative example images of cells expressing YFP-tagged STIM1 wild type, STIM1-F108I, STIM1-H109N, STIM1-H109R, or STIM1-I115F under resting-state conditions and after treatment with $1 \mu \mathrm{M}$ thapsigargin (TG) for $5 \mathrm{~min}$. Scale bar $10 \mu \mathrm{m}$. (B) Mean value of STIM1 cluster formation per cell during resting cell conditions for wild type STIM1, STIM1-F108I, STIM1-H109N, STIM1-H109R, and STIM1-I115F ( $n=22$ to 39 cells per mutant). An asterisk $\left(^{*}\right)$ indicates a significant difference in the number of STIM1 clusters between STIM1 mutants and STIM1 wild type in the resting state $(t$-test: $p<0.01)$ ). (C) Time course of whole-cell patch-clamp average currents at $-86 \mathrm{mV}$ from cells overexpressing STIM1 wild type, STIM1-F108I, STIM1-H109N, STIM1-H109R, and STIM1-I115F. Intracellular solution containing $20 \mathrm{mM}$ EDTA passively induced store depletion. (D) Mean currents after whole-cell break-in ( $n=7$ to 12 cells per mutant, data were taken from three independent transfections). An asterisk $\left(^{*}\right)$ indicates a significant difference in whole-cell break-in currents between STIM1 mutants and STIM1 wild type in the resting state $(t$-test: $p<0.01)$. (E) Representative current/voltage relationships according to whole-cell patch-clamp experiments with cells overexpressing STIM1 wild type, STIM1-F108I, STIM1-H109N, STIM1-H109R, and STIM1-I115F, respectively. 
$\mathrm{I}_{\mathrm{CRAC}}$ is strictly required to stimulate nuclear translocation and thus activate NFAT [31,32]. We have previously overexpressed STIM1-F108I, H109N, H109R, and I115F mutants individually and determined a preferentially higher nuclear localization of NFAT under resting $\mathrm{ER} \mathrm{Ca}^{2+}$ conditions than for wild type STIM1 $[15,33]$. This NFAT translocation screen has offered an effective approach to identifying constitutively active STIM1 mutants [15]. Increased intracellular $\mathrm{Ca}^{2+}$ concentration due to $\mathrm{I}_{\mathrm{CRAC}}$ is directly linked to NFAT-regulated transcriptional activity which is controlled by the $\mathrm{Ca}^{2+}$ - and Calmodulin-dependent phosphatase Calcineurin [33,51,52].

\subsection{Puncta Formation and Constitutive $I_{C R A C}$ Currents Are Induced by STIM1 Hydrophobic Pocket TAM Mutations}

In a remarkably similar activation mechanism, the $\mathrm{Ca}^{2+} /$ Calmodulin/Calcineurin signaling pathway dephosphorylates also TFEB, triggering nuclear translocation [41]. We investigated whether heterologous overexpression of YFP-tagged STIM1 F108I, H109N, H109R, or I115F mutants in HEK cells results in preferential nuclear trans-localization of CFP-tagged TFEB and MITF one day after co-transfection. In fact, this set of constitutively active STIM1 mutants exhibited a significant increase both in nuclear MITF (40-55\% of cells) (Figure 4A,B, Figure S2A) and TFEB (50-65\% of cells) localization (Figure 4C,D, Figure S2B) compared to cells overexpressing STIM1 wild type (MITF: 25\%; TFEB: 30\% of cells).
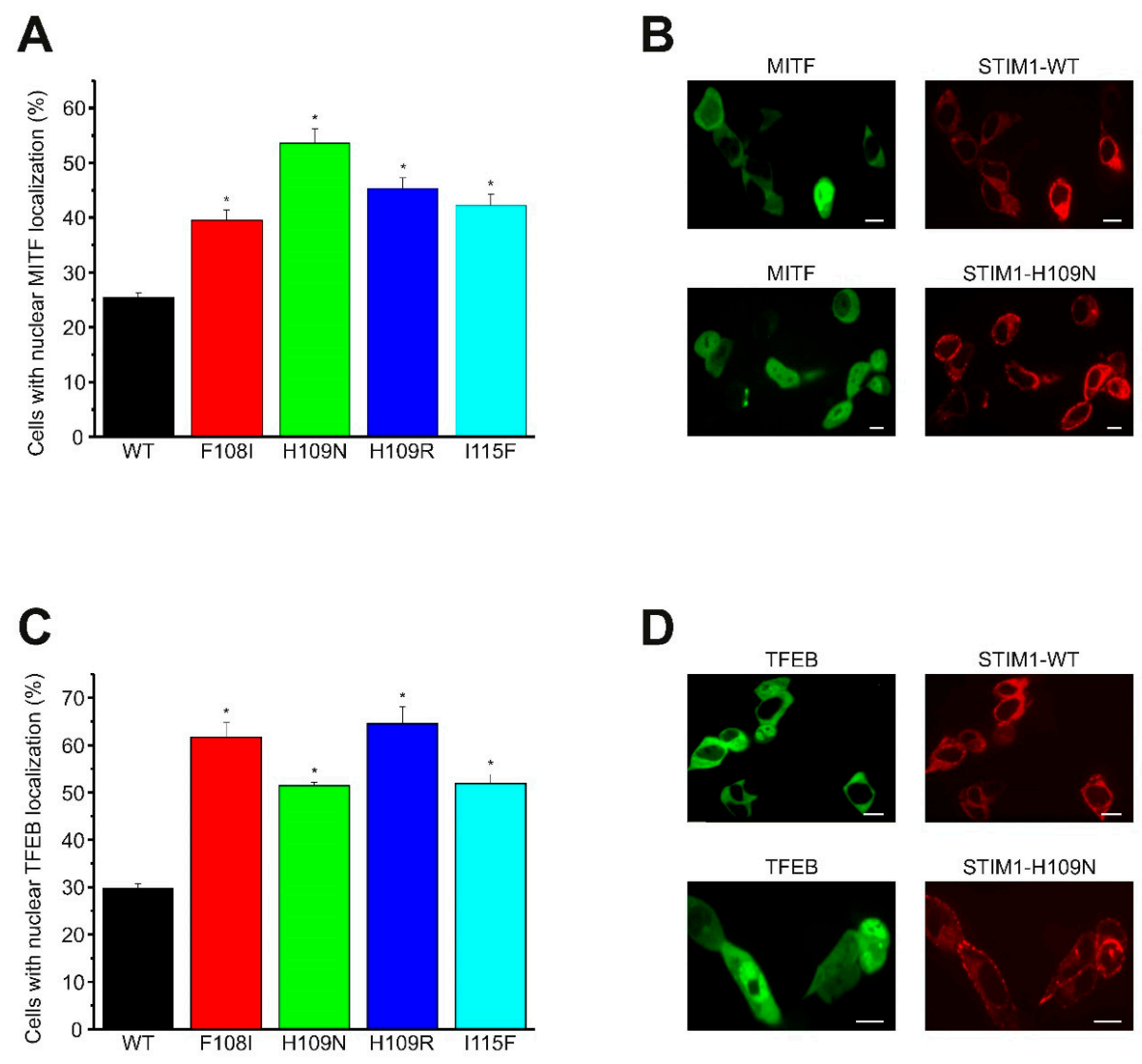

Figure 4. Nuclear trans-localization of microphthalmia-associated transcription factor (MITF)/TFE transcription factor family induced by heterologous expression of non-canonical EF-hand mutants. $(\mathrm{A}, \mathrm{C})$ Statistical analysis provided the percentages of cells with nuclear localization of (A) CFP-tagged MITF or (C) CFP-tagged transcription factor EB (TFEB) upon co-expression with STIM1 wild type, STIM1-F108I, STIM1-H109N, STIM1-H109R, and STIM1-I115F, respectively. For each construct, $n=173$ to 323 cells were measured on three different days. An asterisk $\left(^{*}\right)$ indicates a significantly increased number of cells with nuclear MITF or TFEB localization compared to wild type STIM1 co-expression ( $t$-test: $p<0.01)$. (B,D) Representative example images of cells co-expressing (B) CFP-tagged MITF or (D) CFP-tagged TFEB and YFP-tagged STIM1 wild type, and STIM1-H109N, respectively. Scale bar $10 \mu \mathrm{m}$. 
Another $\mathrm{Ca}^{2+}$-regulated autophagic process is the biogenesis of autophagic vesicles [47]. This $\mathrm{Ca}^{2+}$ signaling pathway links the lysosomal $\mathrm{Ca}^{2+}$-release channel TRPML1 via activation of $\mathrm{Ca}^{2+} /$ Calmodulin-dependent protein kinase kinase $\mathrm{B}$ and AMP-activated protein kinase (AMPK), induction of the Beclin1/VPS34 autophagic complex and generation of phosphatidylinositol 3-phosphate (PI3P) to autophagic vesicle biogenesis [47]. We directly investigated autophagosome biogenesis by determining the number of autophagosomes per cell, using the PI3P reporter GFP-2xFYVE (Figure 5) $[41,43,46]$. For these experiments, we expanded our set of constitutively active (non-canonical EF-hand) STIM1 mutants to include canonical EF-hand mutants (H72Q, H72R, N80K, and E87Q) in RBL mast cells. We have previously determined that the latter type of mutant exhibits cluster formation under resting cell conditions and induces high constitutive $\mathrm{Ca}^{2+}$-entry activity [15]. Overexpression of STIM1 canonical (Figure 5C,D, Figure S2C) and non-canonical (Figure 5A,B, Figure S2C) EF-hand mutants together with the 2xFYVE-autophagosome reporter (Figure 5B,D) led to significantly more autophagosomes per cell than overexpression of STIM1 wild type. In contrast, mock transfected cells and overexpression of STIM1 wild type yielded a similar low number of autophagosome-positive vesicles under resting cell conditions (Figure 5A,C).

A

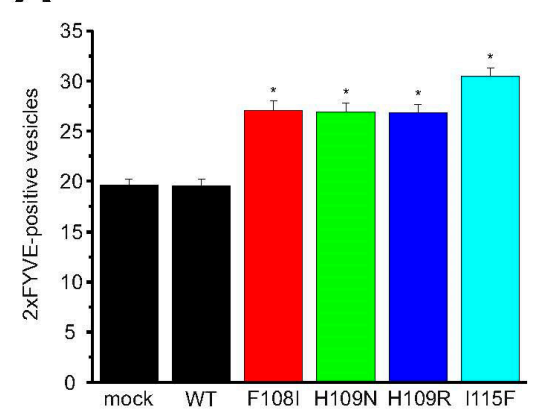

C

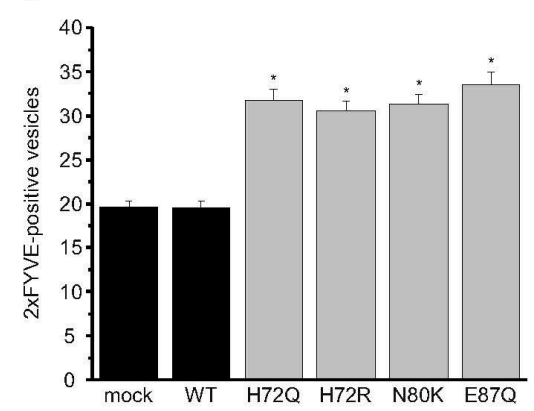

B
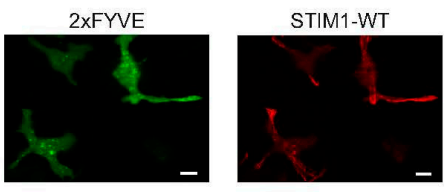

2xFYVE
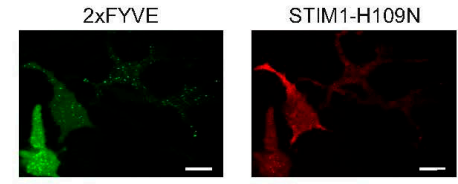

D

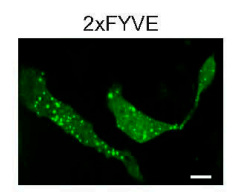

STIM1-H72R

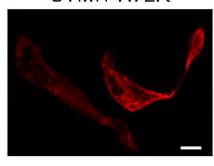

STIM1-E87Q
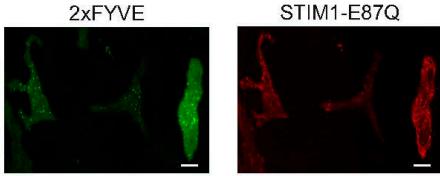

Figure 5. Activation of autophagic pathways by STIM1 canonical and non-canonical EF-hand mutants. (A) Average number of 2xFYVE positive vesicles per cell with overexpression of no STIM1 (mock), STIM1 wild type, STIM1-F108I, STIM1-H109N, STIM1-H109R, and STIM1-I115F, respectively. Each mutant was measured on three different days with $n=66$ to 110 cells. An asterisk (*) indicates a significantly increased number of 2xFYVE positive vesicles found in cells overexpressing STIM1 mutants compared to cells overexpressing STIM1 wild type ( $t$-test: $p<0.01)$. (B) Representative images of cells co-overexpressing lysosomal marker 2xFYVE as well as STIM1 wild type and STIM1-H109N, respectively. Scale bar $10 \mu \mathrm{m}$. (C) Average number of 2xFYVE positive vesicles per cell with overexpression of no STIM1 (mock), STIM1 wild type, STIM1-H72Q, STIM1-H72R, STIM1-N80K, and STIM1-E87Q, respectively. Each mutant was measured on three different days with $n=45$ to 110 cells. An asterisk $\left(^{*}\right)$ indicates a significantly increased number of 2xFYVE positive vesicles found in cells overexpressing STIM1 mutants compared to cells overexpressing STIM1 wild type ( $t$-test: $p<0.01)$. (D) Images representing cells co-overexpressing lysosomal marker 2xFYVE as well as STIM1-H72R and STIM-E87Q, respectively. Scale bar $10 \mu \mathrm{m}$. 
These experiments show that STIM1 mutations that cause tubular aggregate myopathy result in constitutive activity and have a robust stimulatory effect on autophagosome formation and autophagy transcription factors. These mutations affect the non-canonical EF-hand, but do not directly alter $\mathrm{Ca}^{2+}$-ion binding [11,17]. Rather, they destabilize the hydrophobic pocket, which is an essential step in the activation of STIM1.

\section{Discussion}

High-resolution structures in combination with live-cell experiments have demonstrated the importance of luminal STIM1 domains, especially of the canonical and non-canonical EF-hands and the SAM domain, in maintaining a tightly packed protein conformation under resting cell conditions $[10,11,17,18,53]$. Lowering the $\mathrm{Ca}^{2+}$ concentration below resting ER conditions leads to protein destabilization that is induced by unfolding events primarily in the canonical and non-canonical EF-hands $[11,17,34]$. This partially unfolded STIM1 preferentially forms dimers in the initial step of a precisely coordinated activation cascade that leads to gating of SOCE across the plasma membrane. Our study provides new insights into this structural unfolding process upon $\mathrm{Ca}^{2+}$ depletion, especially regarding the non-canonical EF-hand. We combined functional live-cell recordings with MD simulations to reveal the importance of the non-canonical EF-hand in maintaining a hydrophobic pocket. Mutations within this domain have previously been identified in patients suffering from TAM (F108I, H109N, H109R, I115F) [35,36]. In line with previous results, all four TAM mutants investigated resulted in constitutive STIM1 activation and dimerization/oligomerization of STIM1 proteins [15,35,36]. Our MD results showed that all hydrophobic pocket residues are involved in the unfolding process upon $\mathrm{Ca}^{2+}$ depletion. This structural rearrangement is most pronounced for the canonical EF-hand residues L92 and L96. Interestingly, a L92P mutation has been identified in cancer patients, and L92V and L96V mutations, which cause constitutive SOC entry, have been found in patients suffering from tubular aggregate myopathy $[15,35,36,54]$.

Structural analysis of STIM1 in its resting state has shown a configuration largely similar to that of the ubiquitously expressed $\mathrm{Ca}^{2+}$-binding protein Calmodulin [55]. The two EF-hands of STIM1 are wrapped around the SAM domain in a way that closely resembles the configuration of a lobe of Calmodulin in its $\mathrm{Ca}^{2+}$-bound state around its target structures [55]. Interestingly, two residues in the non-canonical EF-hand domain show clear functional differences: In a classical EF-hand (e.g., in Calmodulin), the negatively charged side-chains of the STIM1 F108 and G110 positions would contribute to $\mathrm{Ca}^{2+}$-binding [11]. Engineered mutations (F108D-G110D) lead to STIM1 dimerization/oligomerization instead [11]. These results clearly highlight that the function of these two residues has adapted to the relatively high $\mathrm{Ca}^{2+}$ concentration of the ER under resting conditions. This accords with biochemical experiments that have shown the STIM1-F108I mutant to be less temperature-stable and to have reduced $\mathrm{Ca}^{2+}$ affinity $[11,15]$. Results in this research visualize the unfolding of helix 3 of the non-canonical EF-hand and destabilization of the hydrophobic pocket. Consequently, STIM1-F108I yields maximally activated I $\mathrm{CRAC}_{\mathrm{C}}$ currents, which underlines the importance of the hydrophobic residue in this position. We expect similar structural effects also for other non-canonical EF-hand TAM mutants. As example, the substitution of H109 to more hydrophilic side-chains would no longer favor an orientation of the mutated arginine or asparagine in the hydrophobic pocket and hence would destabilize this pocket.

Destabilization of the luminal STIM1 domain is a starting point for dimerization, which ultimately leads to activation of SOCE. Our study showed that heterologously expressed STIM1 EF-hand mutants exhibit maximally constitutively active SOCE channels, which causes unbalanced intracellular $\mathrm{Ca}^{2+}$ concentrations (see Results section and [15]). Subsequent increase in intracellular $\mathrm{Ca}^{2+}$, either induced by SOCE or lysosomal $\mathrm{Ca}^{2+}$-store depletion, was shown to activate signaling cascades associated with autophagic processes [41,43]. Here, we have reported increased nuclear localization of the transcription factors MITF and TFEB in cells expressing TAM-associated STIM1 mutants (F108I, H109N, H109R, I115F). Translocation of TFEB is a key signaling event for stimulating lysosomal biogenesis 
and autophagy [41,43]. Using PI3P reporter 2xFYVE upon co-expression of STIM1 canonical and non-canonical EF-hand domain mutants, we additionally observed enhanced autophagosome biogenesis. Note that gain-of-function STIM1 mutations, including TAM and Stormorken syndrome mutations, result in a broad spectrum of multisystem diseases characterized by muscle weakness, thrombocytopenia, hyposplenism, ichthyosis, dyslexia, moisis, and short stature [54]. Further research is required to properly characterize TAM at the cellular level, and an autophagy phenotype due to constitutively active $\mathrm{Ca}^{2+}$ signaling, as determined in this research, has not yet been investigated in TAM patients.

In summary, our results suggest a putative impaired $\mathrm{Ca}^{2+}$ homeostasis-induced by constitutively active mutations in the two EF-hands of STIM1 - which leads to nuclear translocation of MITF/TFEB and autophagosome formation. Further studies are required to determine (i) whether endogenous SOCE stimulation is sufficient to induce dephosphorylation of the MITF/TFEB transcription factor through the $\mathrm{Ca}^{2+} /$ Calmodulin-dependent phosphatase Calcineurin and (ii) the potential role of increased cytosolic $\mathrm{Ca}^{2+}$ due to SOCE in autophagic vesicle formation.

\section{Materials and Methods}

\subsection{Plasmid-DNA}

STIM1 DNA constructs (accession number NM_003156), with specific point mutations pre-inserted using the QuikChange site-directed mutagenesis kit (StrataGene, San Diego, CA, USA), were cloned into pECFP-C1 and pEYFP-C1 expression vector systems (Clontech, now Takara, Mountain View, CA, USA). Successful mutation and vector-insertion was confirmed by sequence analysis (Eurofins Genomics, Vienna, Austria).

\subsection{Transfection}

TransFectin Lipid Reagent (BioRad, Vienna, Austria) was used for transient transfection of HEK 293 cells. For each YFP-tagged STIM1 construct or mutant, $1 \mu \mathrm{g}$ of plasmid-DNA was used. For subcellular localization studies, $4 \mu \mathrm{g}$ CFP-tagged MITF or $2 \mu \mathrm{g}$ CFP-tagged TFEB plasmid-DNA was co-transfected. Electrophysiology and 2xFYVE vesicle experiments were performed using rat basophilic leukemia (RBL) cells. RBL cells were electroporated with $7 \mu \mathrm{g}$ STIM1 wild type or mutant plasmid DNA, and for 2xFYVE experiments, $7 \mu \mathrm{g} 2 \mathrm{xFYVE-EGFP} \mathrm{was} \mathrm{co-transfected.} \mathrm{Both} \mathrm{cell} \mathrm{types} \mathrm{were} \mathrm{grown} \mathrm{for} 20-24 \mathrm{~h}$ at $37^{\circ} \mathrm{C}$ in a humidity-controlled incubator with $5 \% \mathrm{CO}_{2}$ after transfection/electroporation and regularly tested for mycoplasma contamination.

\subsection{Electrophysiology}

Electrophysiological experiments were performed with RBL cells 20-24 h after electroporation with $7 \mu \mathrm{g}$ STIM1 wild type or STIM1 mutants. Whole-cell recording configuration was used at $21-25^{\circ} \mathrm{C}$, with an $\mathrm{Ag} / \mathrm{AgCl}$ electrode serving as reference electrode. Voltage ramps were applied every $5 \mathrm{~s}$ from a holding potential of $0 \mathrm{mV}$, covering a range of -90 to $90 \mathrm{mV}$ over $1 \mathrm{~s}$. Experiments were performed with bath solution containing $10 \mathrm{mM} \mathrm{Ca}^{2+}$, and store-dependent activation was induced using $20 \mathrm{mM}$ EGTA. Standard extracellular solution consisted of (in mM) $145 \mathrm{NaCl}, 10 \mathrm{HEPES}, 10 \mathrm{CaCl}_{2}, 10$ Glucose, $5 \mathrm{CsCl}$, and $1 \mathrm{MgCl}_{2} ; \mathrm{pH}$ 7.4. Passive store depletion was induced by an internal pipette solution containing (in $\mathrm{mM}$ ) $145 \mathrm{Cs}$ methane sulphonate, $20 \mathrm{EGTA}, 10 \mathrm{HEPES}, 8 \mathrm{NaCl}$, and $3.5 \mathrm{MgCl}_{2} ; \mathrm{pH}$ 7.2. For liquid junction potential correction, resulting from a $\mathrm{Cl}^{-}$-based bath solution and a sulphonate-based pipette solution, $+12 \mathrm{mV}$ were applied. All currents were leak-corrected by subtracting the initial voltage ramps obtained shortly after break-in with no visible current activation from the measured currents. All experiments were conducted on at least three different days. 


\subsection{Confocal Fluorescence Microscopy}

Confocal fluorescence microscopy was performed with both RBL and HEK 293 cells to identify subcellular localization of STIM1 wild type and mutants, MITF/TFEB transcription factor family (in HEK 293 cells) and 2xFYVE vesicle formation (in RBL cells). Transfected HEK and electroporated RBL cells were grown on coverslips for $20-24 \mathrm{~h}$ at $37^{\circ} \mathrm{C}$ in a humidity-controlled incubator with $5 \%$ $\mathrm{CO}_{2}$. For measurement, the coverslips with the cells were washed and transferred into a solution consisting of $140 \mathrm{mM} \mathrm{NaCl}, 5 \mathrm{mM} \mathrm{KCl}, 1 \mathrm{mM} \mathrm{MgCl} 2,2 \mathrm{mM} \mathrm{CaCl}_{2}, 10 \mathrm{mM}$ glucose, and $10 \mathrm{mM}$ HEPES buffer (adjusted to $\mathrm{pH} 7.4$ with $\mathrm{NaOH}$ ).

Fluorescence images of the subcellular localization of MITF/TFEB transcription factors were recorded using a QLC100 Real-Time Confocal System (VisiTech Int., Sunderland, UK) connected to two Photometrics CoolSNAPHQ monochrome cameras (Roper Scientific, Planegg, Germany) and a dual-port adapter (dichroic: 505lp; cyan emission filter: 485/30; yellow emission filter: 535/50; Chroma Technology Corp., Olching, Germany). This system was attached to an Axiovert 200M microscope (Zeiss, Oberkochen, Germany) in conjunction with two diode lasers (445 nm, $515 \mathrm{~nm}$ ) (Visitron Systems, Puchheim, Germany). Visiview 2.1.1 software (Visitron Systems) was used for image acquisition and control of the confocal system. Illumination times for CFP/FRET and YFP images were recorded consecutively with a minimum delay of about $1000 \mathrm{~ms}$. ImageJ was employed for subcellular localization analysis of the transcription factors by means of intensity measurements of the cytosol and nucleus, distinguishing between three different populations with different nucleus/cytosol ratios: inactive $(<0.85)$, homogenous $(0.85-1.15)$, and active $(>1.15)$.

For fluorescence image recording of 2xFYVE vesicle experiments, a CSU-X1 Real-Time Confocal System (Yokogawa Electric Corporation, Vienna, Austria) fitted with two CoolSNAP HQ2 CCD cameras (Photometrics) was used. a dual port adapter (dichroic: 505lp, cyan emission filter: 470/24, yellow emission filter: 535/30, Chroma Technology Corporation) also formed part of the apparatus. All these parts were connected to an Axio Observer Z1 inverted microscope (Zeiss, Oberkochen, Germany) with two diode lasers (445 and $515 \mathrm{~nm}$, Visitron Systems) and placed on a Vision IsoStation anti-vibration table (Newport Corporation, Irvine, CA, USA).

The VisiView software package (V.2.1.4, Visitron Systems) was used for confocal system control and image generation. The images were created and analyzed on a pixel-to-pixel basis by means of a custom-made software integrated into MATLAB (v7.11.0, The MathWorks, Inc.). Visible 2xFYVE positive vesicle spots for 2xFYVE (mock), STIM1 wild type, and STIM1 mutants in the recorded images were counted and averaged. The same approach, additionally using threshold settings in ImageJ, has been applied for STIM1 cluster quantification.

All experiments were performed on three different days at room temperature, and the resulting data are presented as mean \pm SEM (standard error of the mean) for the indicated number of experiments. Unpaired two-sided Student's $t$-tests for comparison of two groups (performed with Origin Pro 2019) were used to determine significant differences $(p<0.01)$.

\subsection{Simulation for Analysis of the Unfolding}

All analyses used simulations created in previous research [15]. The mean distances between the centers of the EF-hands and residues belonging to the hydrophobic pocket were calculated using the last $100 \mathrm{~ns}$ of simulated STIM1 structures. This was done by subtracting the distances obtained in the folded structure from the distances obtained in the unfolded one for each residue. Finally, all distances were normalized with respect to the greatest distance obtained (L92).

Supplementary Materials: Supplementary materials can be found at http://www.mdpi.com/1422-0067/21/12/4410/s1. Figure S1: Conformational rearrangement of the luminal STIM1 F108I mutant. Figure S2: Activation of autophagy and transcriptional pathways associated with disease-causing canonical and non-canonical STIM1 mutants. 
Author Contributions: R.S. (Rainer Schindl) and R.S. (Romana Schober) conceived the ideas, supervised the project, interpreted the data, and wrote the manuscript. M.S. and R.S. (Romana Schober) performed fluorescence experiments and analyzed the data. M.S. prepared the original draft of the paper. A.T. performed patch-clamp experiments. D.B. and T.S. performed structural analysis and data presentation. V.L. and I.F. cloned the constructs. L.W. and I.D. contributed to the study design, revised the manuscript, and supported data analysis together with all other authors. All authors have read and agreed to the published version of the manuscript.

Funding: We gratefully acknowledge the support of the Austrian Science Fund (FWF) through project grants P28701 to R. Schindl, P32075-B and P28872-B27 to I.F., and P27641, P30567, P32851, and project LIT2018-5-SEE-111 from the JKU Linz to I.D. D.B. was supported by the Czech Science Foundation (19-20728Y). L.W. and T.S. received funding from the FWF project grant P28701 and the Medical University of Graz through the PhD Program "Molecular Medicine". L.W. is a recipient of a DOC Fellowship of the Austrian Academy of Sciences at the Institute of Biophysics at the Gottfried Schatz Research Center, Medical University of Graz.

Acknowledgments: Open Access Funding by the Austrian Science Fund (FWF). Access to the National Grid Infrastructure Metacentrum and the computational resources provided are gratefully acknowledged.

Conflicts of Interest: The authors declare no conflict of interest.

\section{Abbreviations}

$\begin{array}{ll}\text { MDPI } & \text { Multidisciplinary Digital Publishing Institute } \\ \text { MITF } & \text { Microphthalmia-associated transcription factor } \\ \text { DOAJ } & \text { Directory of open access journals } \\ \text { TAM } & \text { Tubular aggregate myopathy } \\ \text { SAM } & \text { Sterile alpha motif } \\ \text { TFEB } & \text { Transcription factor EB } \\ \text { ER } & \text { Endoplasmic reticulum } \\ \text { PM } & \text { Plasma membrane } \\ \text { SERCA } & \text { Sarcoplasmic/endoplasmic reticulum calcium ATPase } \\ \text { SOC } & \text { Store-operated Ca }{ }^{2+} \text { channels } \\ \text { SOCE } & \text { Store-operated Ca }{ }^{2+} \text { channels entry } \\ \text { Ca2+ } & \text { Calcium } \\ \text { NFAT } & \text { Nuclear factor of activated T-cells } \\ \text { CaN } & \text { Calcineurin } \\ \text { NLS } & \text { Nuclear localization signal } \\ \text { MD } & \text { Molecular dynamis } \\ \text { TG } & \text { Thapsigargin } \\ \text { RBL } & \text { Rat basophilic leukemia } \\ \text { HEK } & \text { Human embryonic kidney } \\ \text { YFP } & \text { Yellow fluorescent protein } \\ \text { CFP } & \text { Cyan fluorescent protein } \\ \text { PI3P } & \text { phosphatidylinositol 3-phosphate } \\ \text { STIM1 } & \text { Stromal interaction molecule 1 } \\ \text { TRPML1 } & \text { Transient receptor potential mucolipin 1 } \\ \text { AMPK } & \text { AMP-activated protein kinase } \\ & \end{array}$

\section{References}

1. Bonhenry, D.; Schober, R.; Schmidt, T.; Waldherr, L.; Ettrich, R.H.; Schindl, R. Mechanistic insights into the Orai channel by molecular dynamics simulations. Semin Cell Dev. Biol. 2019, 94, 50-58. [CrossRef] [PubMed]

2. Prakriya, M.; Lewis, R.S. Store-Operated Calcium Channels. Physiol. Rev. 2015, 95, 1383-1436. [CrossRef] [PubMed]

3. Parekh, A.B. Store-operated CRAC channels: Function in health and disease. Nat. Rev. Drug Discov. 2010, 9, 399-410. [CrossRef] [PubMed]

4. Schober, R.; Waldherr, L.; Schmidt, T.; Graziani, A.; Stilianu, C.; Legat, L.; Groschner, K.; Schindl, R. STIM1 and Orai1 regulate $\mathrm{Ca}^{2+}$ microdomains for activation of transcription. Mol. Cell Res. 2018, 7, 1079-1091. [CrossRef] [PubMed] 
5. $\quad$ Feske, S.; Gwack, Y.; Prakriya, M.; Srikanth, S.; Puppel, S.H.; Tanasa, B.; Hogan, P.G.; Lewis, R.S.; Daly, M.; Rao, A. A mutation in Orai1 causes immune deficiency by abrogating CRAC channel function. Nature 2006, 441, 179-185. [CrossRef] [PubMed]

6. Liou, J.; Kim, M.L.; Heo, W.D.; Jones, J.T.; Myers, J.W.; Ferrell, J.E., Jr.; Meyer, T. STIM is a Ca ${ }^{2+}$ sensor $^{2}$ essential for $\mathrm{Ca}^{2+}$-store-depletion-triggered $\mathrm{Ca}^{2+}$ influx. Curr. Biol. 2005, 15, 1235-1241. [CrossRef]

7. Roos, J.; DiGregorio, P.J.; Yeromin, A.V.; Ohlsen, K.; Lioudyno, M.; Zhang, S.; Safrina, O.; Kozak, J.A.; Wagner, S.L.; Cahalan, M.D.; et al. STIM1, an essential and conserved component of store-operated Ca ${ }^{2+}$ channel function. J. Cell Biol. 2005, 169, 435-445. [CrossRef]

8. Lis, A.; Peinelt, C.; Beck, A.; Parvez, S.; Monteilh-Zoller, M.; Fleig, A.; Penner, R. CRACM1, CRACM2, and CRACM3 are store-operated $\mathrm{Ca}^{2+}$ channels with distinct functional properties. Curr. Biol. 2007, 17, 794-800. [CrossRef]

9. Parvez, S.; Beck, A.; Peinelt, C.; Soboloff, J.; Lis, A.; Monteilh-Zoller, M.; Gill, D.L.; Fleig, A.; Penner, R. STIM2 protein mediates distinct store-dependent and store-independent modes of CRAC channel activation. FASEB J. 2008, 22, 752-761. [CrossRef]

10. Zhang, S.L.; Yu, Y.; Roos, J.; Kozak, J.A.; Deerinck, T.J.; Ellisman, M.H.; Stauderman, K.A.; Cahalan, M.D. STIM1 is a $\mathrm{Ca}^{2+}$ sensor that activates CRAC channels and migrates from the $\mathrm{Ca}^{2+}$ store to the plasma membrane. Nature 2005, 437, 902-905. [CrossRef]

11. Stathopulos, P.B.; Zheng, L.; Li, G.Y.; Plevin, M.J.; Ikura, M. Structural and mechanistic insights into STIM1-mediated initiation of store-operated calcium entry. Cell 2008, 135, 110-122. [CrossRef] [PubMed]

12. Luik, R.M.; Wang, B.; Prakriya, M.; Wu, M.M.; Lewis, R.S. Oligomerization of STIM1 couples ER calcium depletion to CRAC channel activation. Nature 2008, 454, 538-542. [CrossRef] [PubMed]

13. Bischof, H.; Burgstaller, S.; Waldeck-Weiermair, M.; Rauter, T.; Schinagl, M.; Ramadani-Muja, J.; Graier, W.F.; Malli, R. Live-Cell Imaging of Physiologically Relevant Metal Ions Using Genetically Encoded FRET-Based Probes. Cells 2019, 8, 492. [CrossRef] [PubMed]

14. Brandman, O.; Liou, J.; Park, W.S.; Meyer, T. STIM2 is a feedback regulator that stabilizes basal cytosolic and endoplasmic reticulum $\mathrm{Ca}^{2+}$ levels. Cell 2007, 131, 1327-1339. [CrossRef] [PubMed]

15. Schober, R.; Bonhenry, D.; Lunz, V.; Zhu, J.; Krizova, A.; Frischauf, I.; Fahrner, M.; Zhang, M.; Waldherr, L.; Schmidt, T.; et al. Sequential activation of STIM1 links $\mathrm{Ca}^{2+}$ with luminal domain unfolding. Sci. Signal. 2019, 12, eaax3194. [CrossRef] [PubMed]

16. Gudlur, A.; Zeraik, A.E.; Hirve, N.; Rajanikanth, V.; Bobkov, A.A.; Ma, G.; Zheng, S.; Wang, Y.; Zhou, Y.; Komives, E.A.; et al. Calcium sensing by the STIM1 ER-luminal domain. Nat. Commun. 2018, 9, 4536. [CrossRef]

17. Enomoto, M.; Nishikawa, T.; Back, S.-I.; Ishiyama, N.; Zheng, L.; Stathopulos, P.B.; Ikura, M. Coordination of a Single Calcium Ion in the EF-hand Maintains the Off State of the Stromal Interaction Molecule Luminal Domain. J. Mol. Biol. 2020, 432, 367-383. [CrossRef]

18. Wu, M.M.; Buchanan, J.; Luik, R.M.; Lewis, R.S. $\mathrm{Ca}^{2+}$ store depletion causes STIM1 to accumulate in ER regions closely associated with the plasma membrane. J. Cell Biol. 2006, 174, 803-813. [CrossRef]

19. Fahrner, M.; Muik, M.; Schindl, R.; Butorac, C.; Stathopulos, P.; Zheng, L.; Jardin, I.; Ikura, M.; Romanin, C. A coiled-coil clamp controls both conformation and clustering of stromal interaction molecule 1 (STIM1). J. Biol. Chem. 2014, 289, 33231-33244. [CrossRef]

20. Muik, M.; Fahrner, M.; Schindl, R.; Stathopulos, P.; Frischauf, I.; Derler, I.; Plenk, P.; Lackner, B.; Groschner, K.; Ikura, M.; et al. STIM1 couples to ORAI1 via an intramolecular transition into an extended conformation. Embo J. 2011, 30, 1678-1689. [CrossRef]

21. Huang, G.N.; Zeng, W.; Kim, J.Y.; Yuan, J.P.; Han, L.; Muallem, S.; Worley, P.F. STIM1 carboxyl-terminus activates native SOC, Icrac and TRPC1 channels. Nat. Cell Biol. 2006, 8, 1003. [CrossRef]

22. Yuan, J.P.; Zeng, W.; Dorwart, M.R.; Choi, Y.J.; Worley, P.F.; Muallem, S. SOAR and the polybasic STIM1 domains gate and regulate Orai channels. Nat. Cell Biol. 2009, 11, 337-343. [CrossRef] [PubMed]

23. Zhou, Y.; Meraner, P.; Kwon, H.T.; Machnes, D.; Oh-hora, M.; Zimmer, J.; Huang, Y.; Stura, A.; Rao, A.; Hogan, P.G. STIM1 gates the store-operated calcium channel ORAI1 in vitro. Nat. Struct. Mol. Biol. 2010, 17, 112-116. [CrossRef]

24. Park, C.Y.; Hoover, P.J.; Mullins, F.M.; Bachhawat, P.; Covington, E.D.; Raunser, S.; Walz, T.; Garcia, K.C.; Dolmetsch, R.E.; Lewis, R.S. STIM1 clusters and activates CRAC channels via direct binding of a cytosolic domain to Orai1. Cell 2009, 136, 876-890. [CrossRef] [PubMed] 
25. Muik, M.; Frischauf, I.; Derler, I.; Fahrner, M.; Bergsmann, J.; Eder, P.; Schindl, R.; Hesch, C.; Polzinger, B.; Fritsch, R.; et al. Dynamic coupling of the putative coiled-coil domain of ORAI1 with STIM1 mediates ORAI1 channel activation. J. Biol. Chem. 2008, 283, 8014-8022. [CrossRef] [PubMed]

26. Frischauf, I.; Muik, M.; Derler, I.; Bergsmann, J.; Fahrner, M.; Schindl, R.; Groschner, K.; Romanin, C. Molecular determinants of the coupling between STIM1 and Orai channels: Differential activation of Orai1-3 channels by a STIM1 coiled-coil mutant. J. Biol. Chem. 2009, 284, 21696-21706. [CrossRef]

27. Stathopulos, P.B.; Schindl, R.; Fahrner, M.; Zheng, L.; Gasmi-Seabrook, G.M.; Muik, M.; Romanin, C.; Ikura, M. STIM1/Orai1 coiled-coil interplay in the regulation of store-operated calcium entry. Nat. Commun. 2013, 4, 2963. [CrossRef] [PubMed]

28. Orci, L.; Ravazzola, M.; Le Coadic, M.; Shen, W.-W.; Demaurex, N.; Cosson, P. STIM1-induced precortical and cortical subdomains of the endoplasmic reticulum. Proc. Natl. Acad. Sci. USA 2009, 106, 19358. [CrossRef]

29. Soboloff, J.; Rothberg, B.S.; Madesh, M.; Gill, D.L. STIM proteins: Dynamic calcium signal transducers. Nat. Rev. Mol. Cell Biol. 2012, 13, 549-565. [CrossRef]

30. Kar, P.; Nelson, C.; Parekh, A.B. CRAC Channels Drive Digital Activation and Provide Analog Control and Synergy to $\mathrm{Ca}^{2+}$-Dependent Gene Regulation. Curr. Biol. 2012, 22, 242-247. [CrossRef]

31. Kar, P.; Parekh, A.B. Distinct spatial $\mathrm{Ca}^{2+}$ signatures selectively activate different NFAT transcription factor isoforms. Mol. Cell 2015, 58, 232-243. [CrossRef] [PubMed]

32. Kar, P.; Samanta, K.; Kramer, H.; Morris, O.; Bakowski, D.; Parekh, A.B. Dynamic Assembly of a Membrane Signaling Complex Enables Selective Activation of NFAT by Orai1. Curr. Biol. 2014, 24, 1361-1368. [CrossRef] [PubMed]

33. Frischauf, I.; Zayats, V.; Deix, M.; Hochreiter, A.; Jardin, I.; Muik, M.; Lackner, B.; Svobodova, B.; Pammer, T.; Litvinukova, M.; et al. A calcium-accumulating region, $\mathrm{CAR}$, in the channel Orai1 enhances $\mathrm{Ca}\left({ }^{2+}\right)$ permeation and SOCE-induced gene transcription. Sci. Signal. 2015, 8, ra131. [CrossRef] [PubMed]

34. Zheng, L.; Stathopulos, P.B.; Schindl, R.; Li, G.Y.; Romanin, C.; Ikura, M. Auto-inhibitory role of the EF-SAM domain of STIM proteins in store-operated calcium entry. Proc. Natl. Acad. Sci. USA 2011, 108, 1337-1342. [CrossRef]

35. Bohm, J.; Chevessier, F.; Koch, C.; Peche, G.A.; Mora, M.; Morandi, L.; Pasanisi, B.; Moroni, I.; Tasca, G.; Fattori, F.; et al. Clinical, histological and genetic characterisation of patients with tubular aggregate myopathy caused by mutations in STIM1. J. Med. Genet. 2014, 51, 824-833. [CrossRef]

36. Bohm, J.; Chevessier, F.; Maues De Paula, A.; Koch, C.; Attarian, S.; Feger, C.; Hantai, D.; Laforet, P.; Ghorab, K.; Vallat, J.M.; et al. Constitutive activation of the calcium sensor STIM1 causes tubular-aggregate myopathy. Am. J. Hum. Genet. 2013, 92, 271-278. [CrossRef]

37. Sardiello, M.; Palmieri, M.; di Ronza, A.; Medina, D.; Valenza, M.; Gennarino, V.; Di Malta, C.; Donaudy, F.; Embrione, V.; Polishchuk, R.; et al. A gene network regulating lysosomal biogenesis and function. Science 2009, 325, 473-477. [CrossRef]

38. Settembre, C.; Di Malta, C.; Polito, V.A.; Garcia Arencibia, M.; Vetrini, F.; Erdin, S.; Erdin, S.U.; Huynh, T.; Medina, D.; Colella, P.; et al. TFEB links autophagy to lysosomal biogenesis. Science 2011, 332, 1429-1433. [CrossRef]

39. Martina, J.A.; Diab, H.I.; Brady, O.A.; Puertollano, R. TFEB and TFE3 are novel components of the integrated stress response. Embo J. 2016, 35, 479-495. [CrossRef]

40. Nezich, C.L.; Wang, C.; Fogel, A.I.; Youle, R.J. MiT/TFE transcription factors are activated during mitophagy downstream of Parkin and Atg5. J. Cell Biol. 2015, 210, 435-450. [CrossRef]

41. Medina, D.L.; Di Paola, S.; Peluso, I.; Armani, A.; De Stefani, D.; Venditti, R.; Montefusco, S.; Scotto-Rosato, A.; Prezioso, C.; Forrester, A.; et al. Lysosomal calcium signalling regulates autophagy through calcineurin and TFEB. Nat. Cell Biol. 2015, 17, 288-299. [CrossRef] [PubMed]

42. Napolitano, G.; Ballabio, A. TFEB at a glance. J. Cell Sci. 2016, 129, 2475-2481. [CrossRef] [PubMed]

43. Zhu, Z.-D.; Yu, T.; Liu, H.-J.; Jin, J.; He, J. SOCE induced calcium overload regulates autophagy in acute pancreatitis via calcineurin activation. Cell Death Dis. 2018, 9, 50. [CrossRef] [PubMed]

44. Frischauf, I.; Litvinukova, M.; Schober, R.; Zayats, V.; Svobodova, B.; Bonhenry, D.; Lunz, V.; Cappello, S.; Tociu, L.; Reha, D.; et al. Transmembrane helix connectivity in Orai1 controls two gates for calcium-dependent transcription. Sci. Signal. 2017, 10, eaao0358. [CrossRef] [PubMed] 
45. Bronisz, A.; Sharma, S.M.; Hu, R.; Godlewski, J.; Tzivion, G.; Mansky, K.C.; Ostrowski, M.C. Microphthalmia-associated transcription factor interactions with 14-3-3 modulate differentiation of committed myeloid precursors. Mol. Biol. Cell 2006, 17, 3897-3906. [CrossRef]

46. Pattni, K.; Jepson, M.; Stenmark, H.; Banting, G. A PtdIns(3)P-specific probe cycles on and off host cell membranes during Salmonella invasion of mammalian cells. Curr. Biol. 2001, 11, 1636-1642. [CrossRef]

47. Scotto Rosato, A.; Montefusco, S.; Soldati, C.; Di Paola, S.; Capuozzo, A.; Monfregola, J.; Polishchuk, E.; Amabile, A.; Grimm, C.; Lombardo, A.; et al. TRPML1 links lysosomal calcium to autophagosome biogenesis through the activation of the CaMKK $\beta / V P S 34$ pathway. Nat. Commun. 2019, 10, 5630. [CrossRef]

48. Honnappa, S.; Gouveia, S.M.; Weisbrich, A.; Damberger, F.F.; Bhavesh, N.S.; Jawhari, H.; Grigoriev, I.; van Rijssel, F.J.; Buey, R.M.; Lawera, A.; et al. An EB1-binding motif acts as a microtubule tip localization signal. Cell 2009, 138, 366-376. [CrossRef]

49. Hoth, M.; Penner, R. Depletion of intracellular calcium stores activates a calcium current in mast cells. Nature 1992, 355, 353-356. [CrossRef]

50. Muik, M.; Fahrner, M.; Derler, I.; Schindl, R.; Bergsmann, J.; Frischauf, I.; Groschner, K.; Romanin, C. A Cytosolic Homomerization and a Modulatory Domain within STIM1 C Terminus Determine Coupling to ORAI1 Channels. J. Biol. Chem. 2009, 284, 8421-8426. [CrossRef]

51. McCaffrey, P.G.; Luo, C.; Kerppola, T.K.; Jain, J.; Badalian, T.M.; Ho, A.M.; Burgeon, E.; Lane, W.S.; Lambert, J.N.; Curran, T.; et al. Isolation of the cyclosporin-sensitive T cell transcription factor NFATp. Science 1993, 262, 750-754. [CrossRef] [PubMed]

52. Luo, C.; Burgeon, E.; Carew, J.A.; McCaffrey, P.G.; Badalian, T.M.; Lane, W.S.; Hogan, P.G.; Rao, A. Recombinant NFAT1 (NFATp) is regulated by calcineurin in T cells and mediates transcription of several cytokine genes. Mol. Cell Biol. 1996, 16, 3955-3966. [CrossRef] [PubMed]

53. Furukawa, Y.; Teraguchi, S.; Ikegami, T.; Dagliyan, O.; Jin, L.; Hall, D.; Dokholyan, N.V.; Namba, K.; Akira, S.; Kurosaki, T.; et al. Intrinsic Disorder Mediates Cooperative Signal Transduction in STIM1. J. Mol. Biol. 2014, 426, 2082-2097. [CrossRef] [PubMed]

54. Morin, G.; Biancalana, V.; Echaniz-Laguna, A.; Noury, J.B.; Lornage, X.; Moggio, M.; Ripolone, M.; Violano, R.; Marcorelles, P.; Marechal, D.; et al. Tubular aggregate myopathy and Stormorken syndrome: Mutation spectrum and genotype/phenotype correlation. Hum. Mutat. 2020, 41, 17-37. [CrossRef] [PubMed]

55. Marshall, C.B.; Nishikawa, T.; Osawa, M.; Stathopulos, P.B.; Ikura, M. Calmodulin and STIM proteins: Two major calcium sensors in the cytoplasm and endoplasmic reticulum. Biochem. Biophys. Res. Commun 2015, 460, 5-21. [CrossRef] 